\title{
Pensamiento de Víctor Andrés Belaunde y José Carlos Mariátegui y su influencia en la formación axiológica de los docentes de la UNAMBA - Perú
}

Víctor Andrés Belaunde and José Carlos Mariátegui's thought and their influence on the axiological training of UNAMBA teachers - Peru

\author{
Oscar Arbieto Mamani* \\ Universidad Nacional Micaela Bastidas de Apurímac - Perú \\ oarbietom@gmail.com \\ Rosmery Sabina Pozo Enciso** \\ Universidad Alas Peruana - Perú \\ mrpozo@gmail.com \\ Yolanda Huillca Durán *** \\ Universidad Alas Peruana - Perú \\ yohuidu@gmail.com
}

\section{RESUMEN}

La investigación explica cómo el conocimiento del pensamiento dos intelectuales peruanos: Víctor Andrés Belaunde y José Carlos Mariátegui influyen en la formación axiológica de los docentes de la Universidad Nacional Micaela Bastidas de Apurímac, el propósito del estudio se orientó en la investigación cuantitativa. Se realizó una encuesta y un cuestionario cerrado a 40 docentes de las Escuelas Académico Profesionales de Educación Inicial y Ciencias Sociales. El resultado demuestra que la percepción de los encuestados se inclina hacia la influencia positiva del pensamiento socialista del autor José Carlos Mariátegui en la labor docente y que al contrario la percepción de influencia del pensamiento de Víctor Andrés Belaunde tiende a ser negativa. Se puede concluir que el pensamiento de José Carlos Mariategui es más conocido y por ende más influyente que el de Víctor Andrés Belaunde en la formación axiológica de los docentes de la UNAMBA.

Palabras Claves: Pensamiento, Conocimiento, Formación axiológica, filosofía, educación.

\section{ABSTRACT}

The investigation explains how the knowledge of the thought of two Peruvian intellectuals: Víctor Andrés Belaunde and José Carlos Mariátegui's influence the axiological training of the teachers of the National University Micaela Bastidas de Apurímac, the purpose of the study was oriented in quantitative research. A survey and a closed questionnaire were carried out with 40 teachers from the Professional Academic Schools of Initial Education and Social Sciences. The result shows that the perception of the respondents leans towards the positive influence of the socialist thought of the author José Carlos Mariátegui in the teaching work and that on the contrary the perception of influence of the thought of Víctor Andrés Belaunde tends to be negative. It can be concluded that José Carlos Mariategui's thinking of is better known and therefore more influential than that of Víctor Andrés Belaunde in the axiological training of UNAMBA teachers.

Keywords: Pensamiento, conocimiento, formación axiológica, filosofía, educación.

\footnotetext{
*Doctor en Educación, Universidad César Vallejo. Magister en Educación (UCV). Profesor ordinario Asociado en la Universidad Nacional Micaela Bastidas de Apurímac.

** Magister en Gerencia Social por la Universidad Alas Peruanas - Lima. Licenciada en Educación con mención en: "Filosofía y Religión” por la Facultad de Teología Pontificia y Civil de Lima - Universidad Católica San José. Docente a tiempo completo. Universidad Alas Peruana, Perú.

*** Magister en Derecho, mención Derecho Civil y Procesal Civil. Docente en Universidad Alas Peruanas
}

Recibido: 05/07/2019 Aceptado: 18/11/2019 


\section{Introducción}

La educación es una herramienta práctica transformadora que se afianza en la reflexión y el manejo del docente. En la actualidad la educación superior debe superar varios obstáculos que no le permiten que el proceso de enseńanza genere un clima de aprendizaje significativo, los retos apuntalan a desarrollar ciudadanos que se sientan comprometidos con su cultura, cosmovisión, valores y además con una visión holística de su entorno social que le lleve a trascender en su contexto histórico, social y cultural.

Las necesidades sociales de las últimas décadas han llevado a plantear nuevos caminos para la formación universitaria, en esta era de la modernidad líquida (Bauman, 2005) resulta necesario determinar nuevas estrategias formativas donde la figura del docente es un elemento valioso, en este no solo recae la labor de formar a sus estudiantes, el profesor es además un actor importante en el sistema de influencias educativas ya que actúa en el proceso de formación de la personalidad profesional, que va más allá del ámbito académico trascendiendo hacia la formación personal y social del joven.

En este sentido, la formación axiológica será un factor esencial en el proceso de enseñanza aprendizaje, pues contempla las principales cualidades y capacidades que un docente debe poseer para poder incluir dentro de la planificación de sus actividades académicas con el propósito de fomentar y dotar a los profesionales de un conjunto de valores morales, éticos, estéticos y espirituales que le preparen para insertarse en el campo laboral y en la vida misma como lo visualiza Soraya Willlen López (2006) cuando insta a que se debe incorporar la formación axiológica al currículo universitario.

Tomando en consideración el panorama antes propuesto en el que se justifica la importancia de la formación axiológica en la enseńanza universitaria, es necesario recalcar que para llegar a esta meta se requiere del manejo de estos elementos por parte de los docentes, para la formación en valores es preciso reflexionar en la vida misma, en el ámbito escolar y en la cultura de su comunidad, a fin de crear una cultura educativa que convierta a un individuo en persona a través de un proceso de humanización y espiritualidad. Es imperante recordar que cada pueblo se convierte en nación al desarrollar sus posibilidades espirituales, y que los valores de un pueblo tienen su origen en la historia, las tradiciones y sus modos de vida (Willen, 2006:49).

Por ende, el presente estudio se enfocó en estudiar a través del paradigma cuantitativo cómo influye el pensamiento de dos grandes eruditos del país como son Víctor Andrés Belaunde el cual se caracterizó por tener una postura filosófica enfocada en la ética del humanismo cristiano personalista, inclinada al análisis de la sociedad, abogando por la educación de todos los ciudadanos, y José Carlos Mariátegui, quien presenta una tendencia hacia el pensamiento socialista-marxista invitando a la lucha de clases, el objetivo principal será conocer cuál de estas dos posturas paradigmáticas es más conocida por los docentes de la UNAMBA, y por tanto cual es la que más influye en su formación axiológica.

\section{El panorama: una sociedad con crisis de valores}

En la actualidad, la humanidad atraviesa por una crisis en la práctica de valores y virtudes, en medio campea la corrupción en distintas esferas de la sociedad, cada día hay más familias disfuncionales; esta crisis axiológica de orden mundial se traduce en la falta de valores y virtudes, en las instituciones educativas a lo largo de nuestra región los estudiantes, docentes, autoridades y personal en general, esta problemática se ve reflejada en la falta de identidad institucional, en la rivalidad entre compañeros de trabajo, coimas a estudiantes por notas, acoso sexual, estas y otras prácticas repudiables se hacen presentes en el día a día educativo . Al respecto Enrique Rojas expone que:

Una vida sin valores queda reducida a un programa cuyo argumento carece de unión, ya que el mesianismo ha desaparecido y los sistemas de redención del hombre-mitos de realización revolucionariase han desvanecido. Sin embargo, sí existe la solidaridad y su consolidación en el hombre actual, que es consciente de su estado de microcosmos, pero que es capaz de unirse con otros en un proyecto común para hacer un mundo mejor (Rojas, 2000: 95).

Muchos autores han debatido sobre los valores, por ser un tema álgido ya que este concepto implica una revisión muy personal que incluye una visión muy subjetiva de la realidad, el mundo de vida ${ }^{1}$ de cada uno es determinante para la formación de los valores, considerando que la realidad es algo que cada uno construye con las experiencias y forman parte de su mundo fenoménico. En otras palabras: el mundo de la vida es un mundo simbólico, mítico y ritual (Mélich, 1998). De igual manera, Scheler al estudiar el tema de los valores, intenta establecer la existencia de principios universales que no pueden aprehenderse como las esencias, por medio de la inteligencia, pero que tienen una función decisiva para la conducta humana puesto que son las bases universales y necesarias de la conducta (Xirau, 2000). Karol Wojtyla (Papa San Juan Pablo II) pasa por alto la discusión entre empiristas e idealistas al tratar de demostrar que en la acción moral, no en la psique o en el cuerpo, hallamos el centro de la persona humana, el núcleo de nuestra humanidad, pues en la acción moral que la mente, el espíritu y el cuerpo llegan a formar la unidad de una persona (Wojtyla, 2011).

De esta manera, la realidad presenta una situación en la que los valores son inmutables, el bien siempre es el mismo, lo que es relativo es el punto de vista hacia el bien o la manera de realizar el bien (Xirau, 2000). En consecuencia se debe un llamado al crecimiento espiritual si se quiere que el hombre light salga de su estado actual en que sólo preocupa el dinero y el placer para evitar las consecuencias típicas que de ello se derivan: tener, acumular, amasar y, por supuesto, ruptura de matrimonio o pareja (una o varias veces) (Rojas, 2000:4). 
Esta realidad plantea un gran desafío, ya que la meta es elevar la calidad de la educación a un nivel más integral, y esto requiere de un docente con una formación axiológica que asuma este reto. Por lo tanto, la enseñanza docente debe estar caracterizada por el aprendizaje para la práctica reflexiva, en la que se tenga por objeto la formación de profesionales con una dimensión más humana que frene la crisis de valores; el docente deberá asumir el compromiso de ser un agente transformador y emancipador, que guie al alumnado hacia la formación de verdaderos valores éticos a través de la adopción de una postura reflexiva, más crítica y transformadora. En definitiva, se trata de conseguir un hombre más digno, que quiere ser más culto para ser más libres; hacer un mundo más cordial y comprensivo; crear un espacio más afectivo, donde quepan lo material, lo espiritual y lo cultural (Rojas, 2000)

\section{Generación del 900: Víctor Andrés Belaunde y Carlos Mariátegui}

Es esta perspectiva el conocer el pensamiento de los intelectuales en la historia del Perú, en este caso Víctor Andrés Belaunde y José Carlos Mariátegui ayudará, a percibir la influencia de sus pensamientos en la formación axiológica, filosófica y educativa de los docentes de la UNAMBA de Apurímac del Perú. Ahora bien, para abordar esta tesis es preciso conocer la realidad de cada uno de los autores, una de las generaciones más articuladas en el ámbito intelectual, fue la del novecientos, también conocida como la generación arielista.

Fueron llamados así por su identificación con Ariel, el libro del uruguayo José Enrique Rodó (1871-1917), los arielistas renovaron el discurso público de su tiempo, apelando al idealismo, al compromiso con la política de sus países y al retorno al nacionalismo programático, reivindicando la historia particular de cada pueblo americano (Santiváñez, 2014: 245)

En cuanto al panorama presente explica Martín Santiváñez que:

La corrupción estaba vinculada a la decadencia materialista de la civilización utilitaria propia del orbe anglosajón. Era un sub- producto, una extensión impuesta por el expansionismo del Calibán. Como remedio, el arielismo propuso una solución mixta que otorgaba a la reforma institucional el apoyo eficaz de una regeneración valorativa. La apuesta por un idealismo panamericano capaz de plasmarse en la construcción de instituciones eficientes, configuraba una ruptura frontal con el clima positivista que dominaba la academia y la administración pública (p. 245).

En Intelectual emblemático de la generación erudita del 900, Víctor Andrés Belaúnde Diez Canseco destacó en la filosofía, las ciencias políticas, la política parlamentaria y la diplomacia. Aportó una preocupación por la religiosidad y la metafísica al positivismo imperante en sus años universitarios (Centro Nacional de Planeamiento Estratégico 2011). Víctor Andrés Belaunde fue un pensador católico, pero el recorrido de su vida empieza por el positivismo y luego tiene una conversión auténtica hacía la iglesia católica estudiando a San Agustín de Hipona y Santo Tomás de Aquino, humanista que plantea un país como esperanza desde el cristianismo.

Su vida política e intelectual se inicia con el discurso titulado La crisis presente, fue una de sus obras más importantes gracias a la repercusión que obtuvo en su época y en el entorno social, será compendio del proyecto ético-institucional arielista, este disertación fue pronunciada por Belaunde el 13 de julio de 1914, en la apertura del año académico del viejo claustro de San Marcos. Es este el joven Belaunde esboza la grave crisis política, económica, social e institucional de la nación peruana además plantea el problema de la crisis moral y ética también, invocando a los valores como instrumento epistemológico para comprender el subdesarrollo y la corrupción de ese «grande y amado enfermo que se llama el Perú» (Centro Nacional de Planeamiento Estratégico, 2011:175).

Belaunde tuvo una visión claramente positiva del país. Según su propuesta, el Perú es una síntesis viviente en la que la cultura andina y la occidental española se habían fusionado creando una nueva civilización. Opinaba que ambas fuentes son buenas, a pesar de las sombras que puedan haber oscurecido ciertas etapas de la historia (Robles, 2013).

El nacionalismo académico y universitario de la generación arielista se convirtió en el caldo de cultivo del posterior indigenismo político de carácter programático- partidista y contribuyó al surgimiento de las diversas izquierdas que, al alcanzar protagonismo, minimizaron la influencia arielista, optando por vincularse intelectualmente al «González-pradismo» y su discurso reivindicador, a manera de pensamiento proto-revolucionario por excelencia. Sánchez, el gran polígrafo aprista que protagonizó, junto a Mariátegui, la polémica sobre el indigenismo, reconoció «la rebeldía general de la promoción de 1920 frente a sus maestros» (Sánchez, 1968, 47)

De este movimiento forma parte José Carlos Mariátegui el cual se estableció como escritor en forma autodidacta. Antes de cumplir 20 años ya era un periodista de opinión afamado que firmaba con el seudónimo de Juan Croniqueur e integraba parte de la bohemia periodística convocada por Abraham Valdelomar. La realidad de José Carlos Mariátegui es vista desde una perspectiva filosófica del marxismo socialista, plantea una visión de Perú desde el lamento, desde la problemática, partidario de la solución marxista. Mariátegui estudia el socialismo que surge en Francia en el siglo XI, posteriormente entrara en contacto con las ideas socialistas de Marx, Mariátegui 
tuvo seguidores por todo el país, sobretodo en la Sierra y Costa del país, todos los partidos de izquierda y socialistas tienen su génesis en el partido mariateguista del Perú.

En la historia del marxismo en América Latina y su relación con los pueblos originarios del continente constituye un capítulo especial que debe referirse con el nombre de socialismo indoamericano la relación entre marxismo y pueblos indios, a fue representado por el ensayista José Carlos Mariátegui (1894-1930). El desarrollo del socialismo indoamericano, justo como expresión latinoamericana del marxismo, significa tratar el marxismo como un simple instrumento metodológico que, liberado de su ropaje teórico europeo, ayuda a analizar la propia realidad. El programa de crear un socialismo indoamericano es un intento de profunda transformación del marxismo vez que ésta influye en él y lo transforma. Conlleva la transformación del marxismo a otro nivel que, siguiendo la terminología del propio Mariátegui, será ideológico, a fin de designar con ello la transformación de las leyes de la dialéctica y de los dogmas del sistema en convicciones personales que dan firmeza en la acción, pero que no conducen al dogmatismo ni a la intolerancia (Dussel, 2009).

En la dimensión filosófica al analizar las obras de ambos autores, en este caso Víctor Andrés Belaunde y José Carlos Mariátegui, subyace una visión ética, que lleva a plantear el OPERARI SEQUITUR ESSE, el obrar sigue al ser; para que se pueda saber cómo debe comportarse el hombre, se debe conocer ¿Qué es el hombre?, estos eruditos proponen un análisis del Perú, y en ella una visión ontológica del hombre, con una ética y un comportamiento embarcados en la perspectiva paradigmática de su formación intelectual y de su propuesta política y social para abordar la crisis imperante en la realidad peruana que le toco vivir.

\section{Planteamiento metodológico}

Para establecer la influencia del conocimiento de la vida y obra de los autores Víctor Andrés Belaunde y José Carlos Mariátegui en la labor docente de la UNAMBA, se aplicó una investigación de tipo cuantitativa, no experimental transversal, en la que se aplicó como instrumento de recolección de datos la encuesta con respuestas cerradas según lo mostrado en la tabla 1.

Tabla 1. Descripción de las preguntas realizadas y rango de valoración

\begin{tabular}{|c|c|c|c|c|c|}
\hline Pregunta & \multicolumn{5}{|c|}{ Descripción } \\
\hline P1 & \multicolumn{5}{|c|}{$\begin{array}{l}\text { ¿En qué nivel ubicaría su conocimiento sobre la vida y obra del escritor Víctor } \\
\text { Andrés Belaunde? }\end{array}$} \\
\hline P2 & \multicolumn{5}{|c|}{$\begin{array}{l}\text { ¿En qué nivel ubicaría su conocimiento sobre la vida y obra del escritor José } \\
\text { Carlos Mariátegui? }\end{array}$} \\
\hline P3 & \multicolumn{5}{|c|}{$\begin{array}{l}\text { ¿En qué nivel considera usted que está influyendo en la labor de los docentes de la } \\
\text { UNAMBA el pensamiento educativo de Víctor Andrés Belaunde? }\end{array}$} \\
\hline P4 & \multicolumn{5}{|c|}{$\begin{array}{l}\text { ¿En qué nivel considera usted que está influyendo en la labor de los docentes de la } \\
\text { UNAMBA el pensamiento educativo de José Carlos Mariátegui? }\end{array}$} \\
\hline P5 & \multicolumn{5}{|c|}{$\begin{array}{l}\text { ¿En qué nivel considera usted que está influyendo en la labor de los docentes de la } \\
\text { UNAMBA el pensamiento filosófico de Víctor Andrés Belaunde? }\end{array}$} \\
\hline P6 & \multicolumn{5}{|c|}{$\begin{array}{l}\text { ¿En qué nivel considera usted que está influyendo en la labor de los docentes de la } \\
\text { UNAMBA el pensamiento filosófico de José Carlos Mariátegui? }\end{array}$} \\
\hline P7 & \multicolumn{5}{|c|}{$\begin{array}{l}\text { ¿En qué nivel considera usted que está influyendo en la labor de los docentes de la } \\
\text { UNAMBA el pensamiento ético humanista de Víctor Andrés Belaunde? }\end{array}$} \\
\hline P8 & \multicolumn{5}{|c|}{$\begin{array}{l}\text { ¿En qué nivel considera usted que está influyendo en la labor de los docentes de la } \\
\text { UNAMBA el pensamiento ético socialista de José Carlos Mariátegui? }\end{array}$} \\
\hline $\begin{array}{l}\text { Rangos de } \\
\text { Valoración }\end{array}$ & $\begin{array}{l}0=\text { Muy } \\
\text { Deficiente }\end{array}$ & $1=$ Deficiente & $2=$ Regular & $3=$ Bueno & 4 = Óptimo \\
\hline
\end{tabular}

Fuente: elaboración propia

La encuesta se aplicó a una muestra total de 40 docentes de la UNAMBA pertenecientes a las especialidades de Educación Intercultural Bilingüe y Ciencia Política y Gobernabilidad de la Facultad de Educación y Ciencias Sociales, como se observa en la tabla 2.

Tabla 2. Distribución de la muestra de estudio

\begin{tabular}{lll}
\hline $\begin{array}{l}\text { FACULTAD DE EDUCACIÓN Y CIENCIAS } \\
\text { SOCIALES }\end{array}$ & $\begin{array}{l}\text { Total } \\
\text { Docentes }\end{array}$ & Porcentaje \\
\hline EAP. Educación Intercultural Bilingüe & 26 & $60 \%$ \\
EAP. Ciencia Política y Gobernabilidad & 14 & $40 \%$ \\
TOTAL & 40 & $100 \%$
\end{tabular}

Fuente: elaboración propia de los autores. 
A partir de los datos obtenidos se realizó estadística descriptiva y gráficos de frecuencia para establecer la percepción de los encuestados respecto a las preguntas de investigación. También se aplicó un análisis de correlación mediante el estadístico R de Pearson para establecer las relaciones o asociaciones entre el conocimiento de la vida y obra de los autores y el ejercicio docente en la UNAMBA, con un grado de significancia de 0,05 y nivel de confianza de 95\%

Luego del tratamiento estadístico de los datos y su respectivo análisis se realizó una discusión cualitativa donde se analizaron las posibles relaciones causales entre las variables propuestas y así identificar y definir cuales aspectos del pensamiento de los autores guardan relación con la labor docente.

Resultados

A continuación, se muestran los resultados obtenidos del tratamiento estadístico de los datos obtenidos a partir de las encuestas realizadas. Se observa en la tabla 3 y la figura 1, la distribución porcentual del nivel de conocimiento de los encuestados respecto a la vida y obra del escritor Víctor Andrés Belaunde (pregunta 1).

Tabla 3. Estadística descriptiva de la pregunta 1

\begin{tabular}{llllll}
\hline Etiqueta & Valor & Frecuencia & Porcentaje & Porcentaje Válido & Porcentaje Acumulado \\
de Valor & 0 & 2 & 5.00 & 5.00 & 5.00 \\
Muy Deficiente & 1 & 9 & 22.50 & 22.50 & 27.50 \\
Deficiente & 2 & 16 & 40.00 & 40.00 & 67.50 \\
Regular & 3 & 9 & 22.50 & 22.50 & 90.00 \\
Bueno & 4 & 4 & 10.00 & 10.00 & 100.00 \\
Óptimo & & 40 & 100.0 & 100.0 & \\
Total & & & & \\
\end{tabular}

Fuente: elaboración propia de los autores.

Figura 1. Distribución de frecuencias de las respuestas a la pregunta 1

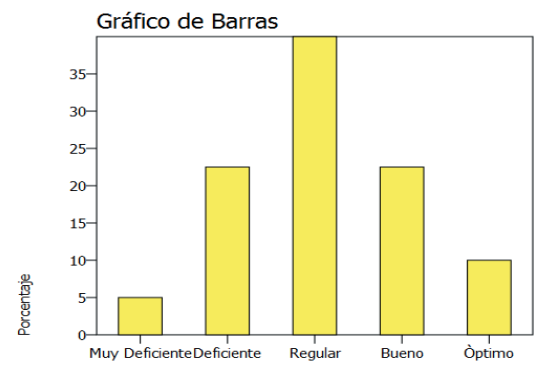

P1

Fuente: elaboración propia de los autores.

Respecto al conocimiento de la vida y obra del autor Víctor Andrés Belaunde, se observó que el 72,5\% de los encuestados manifestó tener un conocimiento de regular a óptimo, siendo el conocimiento regular el de mayor porcentaje con 40,0\%. De lo anterior se deduce que en su mayoría los docentes de la Facultad de Educación y Ciencias Sociales de la UNAMBA tienen cierto conocimiento sobre la vida y obra del citado autor, sin embargo, el nivel de conocimiento se inclina por ser regular, es decir un conocimiento intermedio.

En la tabla 4 y la figura 2 se muestra la distribución de los resultados obtenidos a partir de las encuestas realizadas respecto al nivel de conocimiento de los encuestados sobre la vida y obra del escritor José Carlos Mariátegui (pregunta 2).

Tabla 4. Estadística descriptiva de la pregunta 2

\begin{tabular}{llllll}
\hline $\begin{array}{l}\text { Etiqueta de } \\
\text { Valor }\end{array}$ & Valor & Frecuencia & Porcentaje & $\begin{array}{l}\text { Porcentaje } \\
\text { Válido }\end{array}$ & $\begin{array}{l}\text { Porcentaje } \\
\text { Acumulado }\end{array}$ \\
\hline Deficiente & 1 & 4 & 10.00 & 10.00 & 10.00 \\
Regular & 2 & 7 & 17.50 & 17.50 & 27.50 \\
Bueno & 3 & 17 & 42.50 & 42.50 & 70.00 \\
Óptimo & 4 & 12 & 30.00 & 30.00 & 100.00 \\
Total & & 40 & 100.0 & 100.0 & \\
\hline
\end{tabular}

Fuente: elaboración propia de los autores. 
Figura 2. Distribución de frecuencias de las respuestas a la pregunta 2

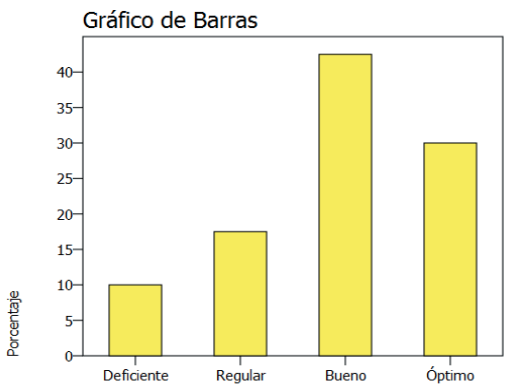

Fuente: elaboración propia de los autores.

En este caso particular, se observó que el 90,0\% de los docentes de la Facultad de Educación y Ciencias Sociales de la UNAMBA tienen conocimiento de regular a óptimo de la vida y obra del autor José Carlos Mariátegui. En la figura 2 se observa una gráfica con sesgo hacia la derecha, lo que indica una percepción de conocimiento que se inclina a ser de buena a óptima, por lo que el nivel de conocimiento bueno fue el de mayor porcentaje con 42,5\%.

La percepción de los encuestados respecto a la influencia del pensamiento educativo de Víctor Andrés Belaunde en la labor docente de los profesores de la Facultad de Educación y Ciencias Sociales de la UNAMBA (pregunta 3), se muestra en la tabla 5 y la figura 3.

\begin{tabular}{llllll}
\hline Etiqueta & Valor & Frecuencia & Porcentaje & $\begin{array}{l}\text { Porcentaje } \\
\text { Válido }\end{array}$ & $\begin{array}{l}\text { Porcentaje } \\
\text { Acumulado }\end{array}$ \\
de Valor & & 6 & 15.00 & 15.00 & 15.00 \\
\hline Muy Deficiente & 0 & 6 & 35.00 & 35.00 & 50.00 \\
Deficiente & 1 & 14 & 42.50 & 42.50 & 92.50 \\
Regular & 2 & 17 & 7.50 & 7.50 & 100.00 \\
Bueno & 3 & 3 & 100.0 & 100.0 & \\
Total & & 40 & & & \\
\hline
\end{tabular}

Tabla 5. Estadística descriptiva de la pregunta 3

Fuente: elaboración propia de los autores.

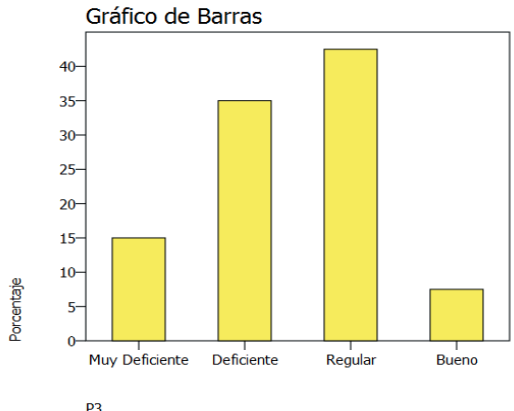

Figura 3. Distribución de frecuencias de las respuestas a la pregunta 3

Fuente: elaboración propia de los autores.

En los resultados se observa que el $92,5 \%$ de los docentes considera que la influencia del pensamiento educativo del autor en la labor docente es de regular a muy deficiente. Lo anterior hace que el gráfico de frecuencias mostrado en la figura 3 se muestre sesgado hacia la izquierda, sin embargo predomina la percepción de la influencia regular con $42,5 \%$. De lo anterior se deduce que la percepción de los encuestados es baja respecto a la influencia del pensamiento educativo de Víctor Andrés Belaunde sobre la labor docente, lo que indica que en su mayoría los profesores consideran que hay poca relación.

En la tabla 6 y la figura 4 se observan los resultados de la estadística descriptiva y la distribución de frecuencias para los resultados de la influencia en la labor de los docentes de la UNAMBA del pensamiento educativo de José Carlos Mariátegui (pregunta 4).

Tabla 6. Estadística descriptiva de la pregunta 4

\begin{tabular}{llll}
\hline Etiqueta & Valor Frecuencia Porcentaje & $\begin{array}{l}\text { Porcentaje } \\
\text { Válido }\end{array}$ & $\begin{array}{l}\text { Porcentaje } \\
\text { Acumulado }\end{array}$ \\
de Valor & & & \\
\hline
\end{tabular}




\begin{tabular}{llllll}
\hline Muy & 0 & 4 & 10.00 & 10.00 & 10.00 \\
Deficiente & 1 & 9 & 22.50 & 22.50 & 32.50 \\
Deficiente & 1 & 14 & 35.00 & 35.00 & 67.50 \\
Regular & 2 & 11 & 27.50 & 27.50 & 95.00 \\
Bueno & 3 & 11 & 5.00 & 5.00 & 100.00 \\
Óptimo & 4 & 2 & 100.0 & 100.0 & \\
Total & & 40 & &
\end{tabular}

Fuente: elaboración propia de los autores.

Figura 4. Distribución de frecuencias de las respuestas a la pregunta 4

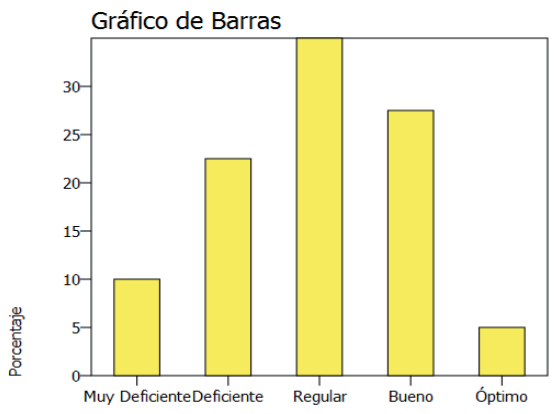

Fuente: elaboración propia de los autores.

Se observa que el 67,5\% considera que la influencia es de regular a óptima, con una percepción regular de 35,0\% y una percepción buena de $27,5 \%$. Lo anterior indica que la mayoría de los encuestados considera que si existe una relación importante entre la labor docente y el conocimiento del pensamiento educativo del autor, lo que hace que el gráfico de frecuencia muestre una tendencia normal bastante simétrica, con predominancia del punto medio, es decir la percepción regular, pero con aporte importante de la percepción buena.

Respecto a la opinión de la muestra encuestada respecto a la influencia en la labor de los docentes de la UNAMBA del pensamiento filosófico de Víctor Andrés Belaunde (pregunta 5), se muestran los resultados en la tabla 7 y la figura 5.

Tabla 7. Estadística descriptiva de la pregunta 5

\begin{tabular}{|c|c|c|c|c|c|}
\hline $\begin{array}{l}\text { Etiqueta } \\
\text { de Valor }\end{array}$ & Valor & Frecuencia & Porcentaje & $\begin{array}{l}\text { Porcentaje } \\
\text { Válido }\end{array}$ & $\begin{array}{l}\text { Porcentaje } \\
\text { Acumulado }\end{array}$ \\
\hline Muy Deficiente & 0 & 7 & 17.50 & 17.50 & 17.50 \\
\hline Deficiente & 1 & 15 & 37.50 & 37.50 & 55.00 \\
\hline Regular & 2 & 11 & 27.50 & 27.50 & 82.50 \\
\hline Bueno & 3 & 6 & 15.00 & 15.00 & 97.50 \\
\hline Óptimo & 4 & 1 & 2.50 & 2.50 & 100.00 \\
\hline Total & & 40 & 100.0 & 100.0 & \\
\hline
\end{tabular}

Fuente: elaboración propia de los autores.

Figura 5. Distribución de frecuencias de las respuestas a la pregunta 5

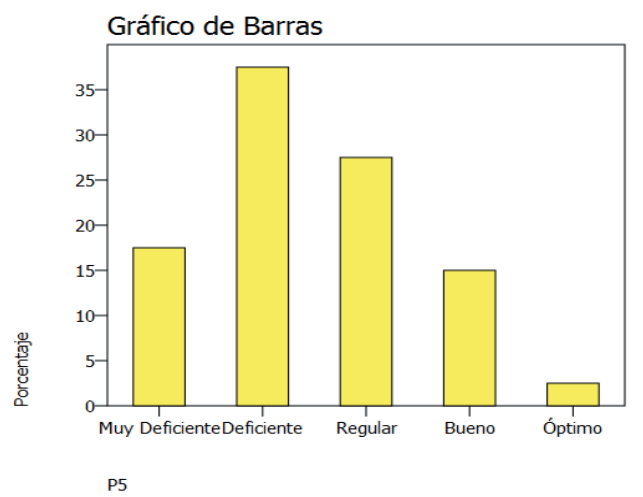

Fuente: elaboración propia de los autores.

Al observar el comportamiento de las respuestas a la pregunta 5 , se obtiene que el $82,5 \%$ de los encuestados considera que la influencia del pensamiento filosófico del autor sobre la labor docente es de regular a muy deficiente, lo que 
implica que la percepción es de poca influencia. Se observa en el gráfico de frecuencias una tendencia sesgada a la izquierda con un valor máximo en la percepción deficiente con 37,5\%, que indica que para la mayor parte de la muestra de estudio, el pensamiento filosófico del autor Víctor Andrés Belaunde no ejerce influencia s obre la labor docente de los profesores de la Facultad de Educación y Ciencias Sociales de la UNAMBA.

En la tabla 8 y la figura 6 se observan los resultados de la estadística descriptiva y la distribución de frecuencias para los resultados obtenidos de la pregunta 6, la cual se refiere a cómo influye en la labor de los docentes de la UNAMBA el pensamiento filosófico de José Carlos Mariátegui.

Tabla 8. Estadística descriptiva de la pregunta 6

\begin{tabular}{|c|c|c|c|c|c|}
\hline $\begin{array}{l}\text { Etiqueta } \\
\text { de Valor }\end{array}$ & Valor & Frecuencia & Porcentaje & $\begin{array}{l}\text { Porcentaje } \\
\text { Válido }\end{array}$ & $\begin{array}{l}\text { Porcentaje } \\
\text { Acumulado }\end{array}$ \\
\hline Muy Deficiente & 0 & 5 & 12.50 & 12.50 & 12.50 \\
\hline Deficiente & 1 & 10 & 25.00 & 25.00 & 37.50 \\
\hline Regular & 2 & 19 & 47.50 & 47.50 & 85.00 \\
\hline Bueno & 3 & 6 & 15.00 & 15.00 & 100.00 \\
\hline Total & & 40 & 100.0 & 100.0 & \\
\hline
\end{tabular}

Fuente: elaboración propia de los autores.

Figura 6. Distribución de frecuencias de las respuestas a la pregunta 6

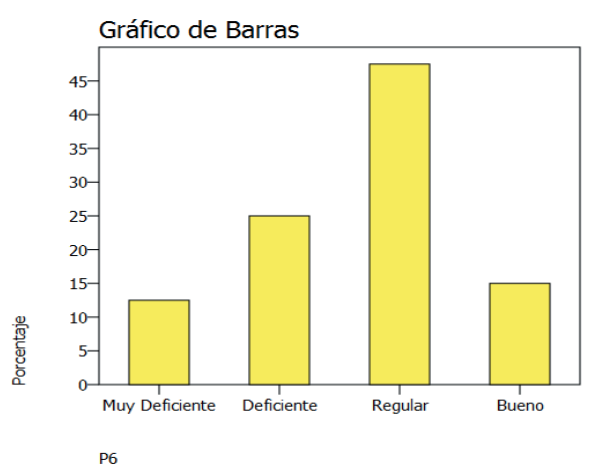

Fuente: elaboración propia del autor.

Se observa una tendencia a que los encuestados tengan una percepción mayoritaria hacia la influencia de regular a buena, ya que el $62,5 \%$ de las respuestas se ubicaron en ese rango. De lo anterior se deduce que la mayoría de la muestra de estudio considera que si existe una influencia del pensamiento filosófico del autor en la labor docente con una percepción regular mayoritaria con $47,5 \%$, lo que se observa en el gráfico de frecuencias, el cual presenta sesgo hacia la derecha.

Respecto a los resultados obtenidos de la opinión sobre la influencia en la labor de los docentes de la UNAMBA del pensamiento ético humanista de Víctor Andrés Belaunde (pregunta 7), se observa la tendencia de los mismos respecto a la estadística descriptiva en la tabla 9 y la distribución de frecuencias en la figura 7.

Tabla 9. Estadística descriptica de la pregunta 7

\begin{tabular}{|c|c|c|c|c|c|}
\hline $\begin{array}{l}\text { Etiqueta } \\
\text { de Valor }\end{array}$ & Valor & Frecuencia & Porcentaje & $\begin{array}{l}\text { Porcentaje } \\
\text { Válido }\end{array}$ & $\begin{array}{l}\text { Porcentaje } \\
\text { Acumulado }\end{array}$ \\
\hline Muy Deficiente & 0 & 4 & 10.00 & 10.00 & 10.00 \\
\hline Deficiente & 1 & 17 & 42.50 & 42.50 & 52.50 \\
\hline Regular & 2 & 17 & 42.50 & 42.50 & 95.00 \\
\hline Bueno & 3 & 2 & 5.00 & 5.00 & 100.00 \\
\hline Total & & 40 & 100.0 & 100.0 & \\
\hline
\end{tabular}

Fuente: elaboración propia de los autores.

Figura 7. Distribución de frecuencias de las respuestas a la pregunta 7 


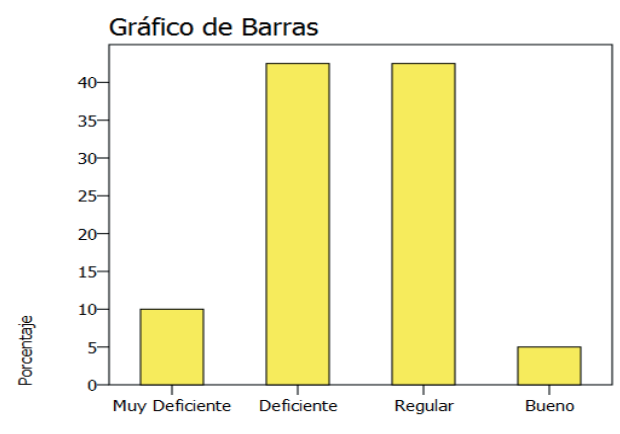

P7

Fuente: elaboración propia de los autores.

Los resultados indican una tendencia bastante uniforme de la percepción de los encuestados respecto a la pregunta 7 , sin embargo el 52,5\% se inclina hacia una influencia de deficiente a muy deficiente, por lo que se puede inferir que la mayoría no considera influyente el pensamiento ético humanista del autor en la labor docente. A pesar de lo anterior, en la figura 7 se observa una distribución centrada con igual frecuencia en las percepciones deficiente y regular, por lo que se podría decir que no es concluyente afirmar una tendencia de las opiniones, pues el margen entre la percepción regular a bueno es pequeño respecto a la percepción de deficiente a muy deficiente.

Las respuestas a la pregunta sobre la influencia en la labor de los docentes de la UNAMBA del pensamiento ético socialista de José Carlos Mariátegui (pregunta 8) se observan en la tabla 10 con el tratamiento estadístico descriptivo correspondiente. También se muestra en el gráfico de la figura 8, la distribución de frecuencias de las opiniones obtenidas.

Tabla 10. Estadística descriptiva de la pregunta 8

\begin{tabular}{llllll}
\hline Etiqueta & Valor & Frecuencia & Porcentaje & $\begin{array}{l}\text { Porcentaje } \\
\text { Válido }\end{array}$ & $\begin{array}{l}\text { Porcentaje } \\
\text { Acumulado }\end{array}$ \\
de Valor & & & & & 15.00 \\
\hline Muy Deficiente & 0 & 6 & 15.00 & 15.00 \\
Deficiente & 1 & 11 & 27.50 & 27.50 & 42.50 \\
Regular & 2 & 14 & 35.00 & 35.00 & 77.50 \\
Bueno & 3 & 9 & 22.50 & 22.50 & 100.00 \\
Total & & 40 & 100.0 & 100.0 & \\
\hline
\end{tabular}

Fuente: elaboración propia de los autores.

Figura 8. Distribución de frecuencias de las respuestas a la pregunta 8

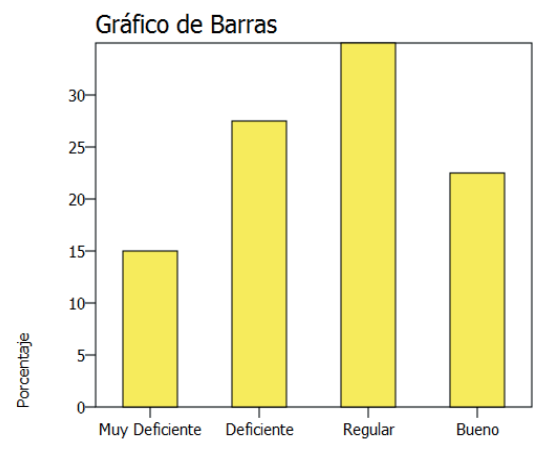

Fuente: elaboración propia de los autores.

Se observa que el $57,5 \%$ de los encuestados considera una influencia de regular a buena del pensamiento ético socialista del autor sobre la labor docente. A pesar de que un porcentaje importante (42,5\%) opina que la influencia es deficiente a muy deficiente, es evidente que existe un sesgo en la percepción que inclina las opiniones hacia una influencia positiva. Lo anterior se puede observar en la figura 8, donde la tendencia de la distribución de frecuencias es sesgada hacia la derecha, es decir hacia las opiniones positivas.

En la tabla 11 se muestra la comparación entre la percepción de los encuestados respecto a la influencia de los pensamiento de los autores Víctor Andrés Belaunde y José Carlos Mariátegui sobre la labor docente de los profesores de la Facultad de Educación y Ciencias Sociales de la UNAMBA. 
Tabla 11. Resumen sobre la influencia del pensamiento de los autores Víctor Andrés Belaunde y José Carlos Mariátegui.

\begin{tabular}{lll}
\hline Pensamiento & Víctor Andrés Belaunde & José Carlos Mariátegui \\
\hline Educativo & $-92,5 \%$ & $+67,5 \%$ \\
Filosófico & $-82,5 \%$ & $+62,5 \%$ \\
Ético Humanista/Ético & $-52,5 \%$ & $+57,5 \%$ \\
Socialista & & \\
\hline
\end{tabular}

Fuente: elaboración propia de los autores.

Se observa en la tabla 11 que en términos generales, la percepción de los encuestados se inclina hacia la influencia positiva (regular a óptima) del pensamiento del autor José Carlos Mariátegui en la labor docente en la Facultad de Educación y Ciencias Sociales de la UNAMBA y que al contrario la percepción de influencia del pensamiento de Víctor Andrés Belaunde tiende a ser negativa (regular a muy deficiente). Lo anterior se correlaciona con el conocimiento de ambas autores por parte de los encuestados, ya que el 90,0\% aseguró tener un conocimiento positivo (regular a óptimo) respecto a la obra de José Carlos Mariátegui y en el caso de Víctor Andrés Belaunde el porcentaje de conocimiento fue también positivo pero menor con $72,5 \%$. Esta relación también se puede sustentar al observar los gráficos de frecuencia mostrados en las figuras 1 y 2, en donde para el caso del conocimiento sobre la obra del autor Víctor Andrés Belaunde la distribución es simétrica con predominio de la media (regular) y en el caso del conocimiento de la obra del autor José Carlos Mariátegui la distribución mostró un marcado sesgo a la derecha con predominio de la opción buena.

A partir de los datos obtenidos, se realizó un análisis de correlación para sustentar la posible relación entre el nivel de conocimiento de los encuestados de las obras de los autores y su percepción de la influencia del pensamiento de cada uno de ellos en la labor docente.

En la tabla 12 se pueden observar los resultados obtenidos del análisis de correlación entre el conocimiento de los encuestados de la obra del escritor Víctor Andrés Belaunde.

Tabla 12. Correlación entre el nivel de conocimiento de la vida y obra del escritor Víctor Andrés Belaunde con la labor docente.

\begin{tabular}{|c|c|c|c|c|c|}
\hline & & P1 & P3 & P5 & P7 \\
\hline \multirow[t]{3}{*}{ P1 } & Correlación de Pearson & 1.00 & .39 & .15 & .14 \\
\hline & Sig. (2-colas) & & .013 & .369 & .379 \\
\hline & $\mathrm{N}$ & 40 & 40 & 40 & 40 \\
\hline
\end{tabular}

Fuente: elaboración propia de los autores.

En la tabla de correlación se observa que para todos los casos existe una relación positiva, es decir con $\mathrm{R}>0$. En el caso de la relación entre el conocimiento y la percepción de influencia de los pensamientos del escritor, las correlaciones fueron según la clasificación de Hopkins (2014), moderada para el pensamiento educativo y bajas para los pensamientos filosóficos y ético humanista. Por otro lado, se observa que sólo la relación entre el conocimiento y el pensamiento educativo es estadísticamente significativa (Sig. < 0,05). Lo anterior permite inferir que, según la opinión de los entrevistados, los pensamientos filosóficos y éticos humanistas del escritor Víctor Andrés Belaunde no guardan relación significativa con la labor docente en la UNAMBA.

Tabla 13. Correlación entre el nivel de conocimiento de la vida y obra del escritor José Carlos Mariátegui con la labor docente.

\begin{tabular}{llllll}
\hline & P2 & P4 & P6 & P8 \\
\hline P2 & Correlación de Pearson & 1.00 & .30 & .24 & .35 \\
& Sig. (2-colas) & & .057 & .133 & .026 \\
& $\mathrm{~N}$ & 40 & 40 & 40 & 40 \\
\hline
\end{tabular}

Fuente: elaboración propia de los autores.

En los resultados mostrados en la tabla 13, se observa que existe correlación positiva $(\mathrm{R}>0)$ entre el conocimiento de los entrevistados de la vida y obra del escritor y el pensamiento del mismo. Según la clasificación de Hopkins (2014) todas las relaciones son moderadas, destacándose la existente entre el conocimiento de los entrevistados y su percepción de la influencia del pensamiento ético socialista del autor en la labor docente con el mayor R de 0,35. En concordancia con lo anteriormente planteado, se observa que sólo la correlación del conocimiento de los encuestados con su opinión sobre el pensamiento ético socialista del escritor es estadísticamente significativa con Sig. $<0,05$.

\section{Conclusiones}

Como resultado se obtuvo que los encuestados se inclinan hacia una influencia positiva (regular a óptima) sobre el pensamiento de José Carlos Mariátegui en la labor docente en la Facultad de Educación y Ciencias Sociales de la UNAMBA y que al contrario la percepción de influencia del pensamiento de Víctor Andrés Belaunde tiende a ser 
negativa (regular a muy deficiente). Por tanto, se puede concluir que el pensamiento de José Carlos Mariátegui es más conocido y por ende más influyente que el de Víctor Andrés Belaunde en la formación axiológica de los docentes de la UNAMBA, demostrando que el pensamiento inclinado al socialismo es el que más predominio en la formación docente.

Este espacio sirve para reflexionar sobre la importancia de concebir el nuevo rol estratégico de la universidad, en función de la realidad apurimeńa y nacional, que impulse nuevos sistemas de gestión estratégica. Sus retos a futuro consisten en introducir cambios significativos en la gestión institucional, en el sistema de enseñanza; convertirse en un centro de formación de ciudadanos comprometidos con su región a través del conocimiento y manejo del pensamiento de intelectuales con posturas filosóficas que fomenten la formación axiológica. La importancia de esta investigación radica en deliberar sobre en el aporte que pueden realizar las instituciones de educación superior si se incorpora a su diseño curricular, por ende, a sus temáticas de clases y a tópicos de las investigaciones aspectos éticos y morales a fin de promover el desarrollo de una cultura de justicia, encaminada hacia el desarrollo de la ética y las virtudes. Sobre todo, en esta era digital donde el conocimiento se encuentra a la mano de todos y de forma inmediata, en la cual se comparte información y rituales sociales que se alejan de las pautas de una adecuada educación en valores.

En tal sentido, se propone la creación de un tribunal de honor o cátedras libres que se encarguen de velar por la organización a través de eventos, actividades extracurriculares y otras acciones que promuevan un ambiente ético, transparente, axiológico, con una cultura de paz y tolerancia que permita la congregación de docentes, estudiantes, autoridades de la institución y demás miembros que hacen vida académica.

De igual manera, se sugiere el diseño de un código de ética profesional de la Universidad Nacional Micaela Bastidas de Apurímac (UNAMBA), con la colaboración y aporte de todo el claustro de académico, a fin de establecer normas sobre el actuar, la moral y la ética del docente con el propósito de garantizar el mejoramiento del acto educativo, el buen desenvolvimiento de la praxis educativa y el fomento de los valores y las buenas costumbres en las aulas de clases y demás espacios del recinto universitario.

Así mismo, se propone la creación de programas de formación y capacitación axiológica para los docentes de la Universidad Nacional Micaela Bastidas de Apurímac, que se sustente en teorías y paradigmas que afiancen la formación en valores, y que sirvan de como centro de discusión donde se generen nuevas propuestas que generen una verdadera transformación no solo en el personal docente sino en todos los grupos que forman parte de la universidad.

Como punto final, se debe recalcar la importancia de establecer correctivos e incentivar la formación axiológica en la institución, y servir de ejemplo para que a nivel regional y nacional otras entidades educativas, gubernamentales, públicas y privadas, puedan incorporar este tipo de estrategias y programas que permitan disminuir un poco la crisis de valores que está acaeciendo en la actualidad y forjar una verdadera transformación social y una cultura de paz.

\section{REFERENCIAS BIBLIOGRÁFICAS}

Bauman, Zygmun. (2005). Los retos de la educación en la modernidad líquida. Barcelona: Editorial Gedisa.

Centro Nacional de Planeamiento Estratégico (2011). Pensadores de la república. Ideas y propuestas vigentes para el Perú del siglo XXI. Perú: Universidad Alas Peruanas.

Dussel, Enrique., Eduardo Mendieta., y Bohóguez. C. (2009). El pensamiento filosófico latinoamericano, del Caribe y "latino" (1300-2000) historia, corrientes, temas y filósofos. México: siglo XXI/ centro de cooperación Regional para la Educación de Adultos en América latina y el Caribe (CREFAL).

Mélich, Joan-Carles. (1998). Antropología simbólica y acción educativa. España: Paidós.

Robles Sotomayor, Fernando Martín (2013). Introducción a las ciencias sociales y económicas. Perú: Universidad Continental.

Rojas, Enrique. (2000). El hombre Light. Una vida sin valores. Argentina: Planeta.

Sánchez, Luis Alberto. (1968). Balance y liquidación del novecientos. ¿'Tuvimos maestros en nuestra América? Lima: Universidad Nacional Mayor de San Marcos.

Vivanco Santiváńez, Martín (2014). «Corrupción y regeneración: Víctor Andrés Belaunde y La crisis presente.» Revista de Indias, nº 260 241-274. Doi:10.3989/revindias.2014.009.

Willen López, Soraya. (2006). Integración de la formación axiológica al curriculum universitario. México: Universidad Autónoma de Baja California.

Wojtyla, Karol. (2011). Persona y Acción. Madrid: Palabra.

Xirau, Ramón. (2000). Introducción a la historia de la filosofía. México: Universidad Nacional Autónoma de México. 